\title{
Endocrine Disruptors: Time to Act
}

\author{
Mariana F. Fernández • Marta Román • \\ Juan Pedro Arrebola $\cdot$ Nicolás Olea
}

Published online: 16 September 2014

(C) Springer International Publishing AG 2014

\begin{abstract}
Recent decades have seen progress in the identification and quantification of a wide array of chemicals with endocrine-active properties. Exposure to these so-called endocrine-disrupting chemicals (EDCs) has been implicated in an increase in certain adverse health effects, and some new prospective birth cohort studies have yielded suggestive results on these exposure-effect relationships. Major research efforts have focused on the EDC exposure of women of childbearing age, because of concerns about embryonic and fetal susceptibility to these chemicals. Investigations have shown that mothers and children are exposed to a complex mixture of compounds; therefore, studies on the health impact of EDC exposure should not be limited to the individual effects of single agents but should rather consider the cumulative effects of multiple chemicals. There is considerable political debate about the need for measures to reduce or avoid exposure to EDCs. While a tighter regulation of exposure to EDCs is being implemented, health professionals and public health practitioners should acquire knowledge of the problem, recognize exposure, and warn the general population about the health risks.
\end{abstract}

Keywords Endocrine disruptors $\cdot$ Adverse effects $\cdot$ Risk assessment $\cdot$ Prenatal exposure

\footnotetext{
M. F. Fernández $\cdot$ M. Román $\cdot$ N. Olea

Department of Radiology, University of Granada, Granada, Spain

M. F. Fernández $(\bowtie) \cdot J$ J. P. Arrebola $\cdot$ N. Olea Instituto de Investigación Biosanitaria ibs.GRANADA, University of Granada, San Cecilio University Hospital, Av. Madrid s/n, Granada 18071, Spain

e-mail:marieta@ugr.es
}

M. F. Fernández $\cdot$ J. P. Arrebola • N. Olea

CIBER Epidemiología y Salud Pública (CIBERESP), Madrid, Spain

\section{Introduction}

The ever-growing use of chemicals in modern society has resulted in their increasing production and use, reaching global dimensions. Some chemicals, such as pesticides, are intentionally released into the environment, while others are released as the result of accidents and spills. Chemicals that are the by-products of manufacturing processes can also enter the environment through various pathways. Over recent decades, observations of the effects of exposure to some anthropogenic chemicals, mainly on wildlife, have increased public awareness of the risks they pose [1].

According to a recent update from the World Health Organization (WHO) (2013) [2], endocrine-disrupting chemicals (EDCs) are defined as chemicals with the capacity to modify hormonal balance and embryonic development and to promote adverse health effects on living organisms or on their offspring. EDCs are highly diverse, with different origins, structures, and functions [3]. They can interfere with the endocrine system by mimicking the action of naturally produced hormones; by preventing the action of endogenous hormones; by altering the synthesis and function of hormone receptors; or by modifying the synthesis, transport, metabolism, or excretion of hormones [4]. They can interfere with the effects of endogenous hormones by acting as receptor agonists or antagonists (or both, when acting as modulators), altering hormonal signaling and therefore the action of hormones. EDCs can also affect hormone concentrations indirectly by acting on signaling pathways that control hormone production or elimination $[2,3]$. Finally, there is increasing scientific evidence that EDCs can affect the exposed organism, its offspring, and future generations through non-genomic modifications and/or epigenetic changes [5]. 


\section{Human Exposure to EDCs}

Biomonitoring studies have shown that humans are exposed to hundreds of EDCs. The lipophilicity and resistance to degradation of some EDCs, e.g., organochlorine pesticides or polybrominated diphenyl ethers (used as flame retardants), means that they accumulate in the fatty tissues of organisms after their release into the environment. Other EDCs to which humans are exposed daily, e.g., bisphenol A (from polycarbonate plastics), phthalates (softener in plastics), or some organophosphate pesticides, are rapidly metabolized and excreted but also contribute to the internal dose [4].

The impact of EDC exposure on human health strongly depends on the age at which it occurs. Thus, the effects of exposure in utero or during pre-puberty can be expected to differ from those of exposure during adulthood. Both embryos and neonates are highly sensitive to EDC exposure and suffer more severe adverse effects than do adults [6, 7]. Thus, exposure during development to EDCs at low doses can produce functional changes in gene expression and may lead to an increased risk of dysfunction and disease later in life, despite the absence of phenotypic changes observable at birth [5]. Many EDCs can cross the placenta and enter the fetus during a highly critical window of vulnerability to their adverse effects [6-8]. Hence, the fetus can be exposed not only to persistent and bio-accumulated substances stored in the mother's adipose tissue and mobilized during pregnancy but also to widespread EDCs [9, 10]. EDCs can also reach the newborn via maternal breast milk [11].

The endocrine disruption hypothesis fits well the paradigm of the fetal origin of disease, which suggests that interactions between the developing organism and the environment determine the risk of disease in adulthood. The original concept of the "fetal basis of adult disease" was changed to the "developmental origin of adult disease" and most recently to the "developmental origins of health and disease (DOHaD)", given that adverse effects may emerge in childhood and adolescence, and health-disease represents a continuous spectrum of outcomes in response to risk factors [12].

The chemical analysis of human placentas has been proposed as an ideal method to investigate exposure of the mother-infant pair to EDCs, yielding data on the exposure of both mother and fetus with no need for an invasive procedure [13].

\section{Prenatal Exposure to EDCs}

Pregnancy is the time when exposure to EDCs can disrupt or interfere with the physiology of developing cells, tissues, and/ or organs, leading to permanent adverse health effects to the exposed organism and future generations [14, 15]. Several population-based studies have found that virtually all pregnant women had measurable levels of different EDCs in their bodies [14-16]. Published data on EDC exposure during pregnancy have verified the presence of numerous chemical residues in different female human tissues. For example, in a population-based cohort study in Southern Spain that focused on prenatal environmental exposures in relation to growth, development, and health from early fetal life until childhood [14], organochlorine compounds (OCs), polychlorobiphenyls (PCBs), dioxins, furans, benzophenones, parabens, and bisphenols, among others, were present in placenta tissue [9, 10, 17-21].

Thus, the presence of 17 OCs (o,p'-DDT; p,p'-DDT; o, $p^{\prime}$ DDD; p,p'-DDE; endosulfan-I and -II; endosulfan-diol, -sulfate, -lactone, and -ether; aldrin; dieldrin; endrin; lindane; hexachlorobenzene [HCB]; mirex and methoxychlor) was investigated in the INMA (INfancia y Medio Ambiente [Environment and Childhood] project) cohort. All placentas studied $(n=311)$ were positive for at least one OC, with a mean of eight residues per placenta (range 2-15 compounds). The most frequent compound was p,p'-DDE (96.2\%), with a mean value of $9.21 \mathrm{ng} / \mathrm{g}$ of placenta, followed by lindane $(76.4 \%)$, endosulfan-diol (63.2\%), o,p'-DDT (55.9\%), endosulfan-I $(52.7 \%)$, and endosulfan-ether $(50.0 \%)$. Around $98 \%$ and $96 \%$ of the samples, respectively, had detectable levels of at least one of the studied DDT and endosulfan isomers/ metabolites [9, 17]. Further, more than half of the placentas $(58.5 \% ; 182 / 311)$ were found to contain free bisphenol A (BPA) at concentrations ranging from 0.5 to $134.0 \mathrm{ng} / \mathrm{g}$ of placenta, suggesting exposure of the fetus to this EDC. BPA was also detected in the urine of mothers during the third trimester of pregnancy and in the urine of their children at the age of 4 years [10].

Exposure to four parabens and six benzophenones was also investigated in human placental tissue samples [18, 19]. Methyl-paraben was detected in $92 \%$ (286/311) of analyzed samples and quantified in $91 \%$ (283/311), at concentrations ranging from 0.1 to $22.1 \mathrm{ng} / \mathrm{g}$ placenta; ethyl-paraben was detected in $70.7 \%(220 / 311)$ of the samples and quantified in $59.2 \%(184 / 311)$ at concentrations of $0.2-12.8 \mathrm{ng} / \mathrm{g}$ placenta; propyl-paraben was detected in $78.1 \%$ (243/311) of the samples and quantified in $70.1 \%(218 / 311)$ at concentrations of $0.2-2.7 \mathrm{ng} / \mathrm{g}$ placenta; and butyl-paraben was detected in $30.2 \%(94 / 311)$ of the samples and quantified in $18.3 \%(57 /$ 311 ), at concentrations ranging from 0.2 to $1.6 \mathrm{ng} / \mathrm{g}$ placenta [20]. Among the six benzophenones investigated, benzophenone-1, benzophenone-2, benzophenone-3, and benzophenone- 8 were not detected in any of the placenta samples; 4-hydroxybenzophenone was detected in $49.8 \%$ (155/311) and quantified in $47.6 \%$ (148/311) of the samples, at concentrations ranging from 0.2 to $5.3 \mathrm{ng} / \mathrm{g}$ placenta; and benzophenone-6 was detected in $12.5 \%$ (39/311) of the samples and quantified in $11.3 \%(35 / 311)$, at concentrations ranging from 0.4 to $40.6 \mathrm{ng} / \mathrm{g}$ placenta [20]. 
Finally, the concentration of seven polychlorinated dibenzo-p-dioxins (PCDDs), ten dibenzofurans (PCDFs), four non-ortho PCBs (PCB-77, -81, -126, and -169), eight monoortho PCBs (PCB-105, -114, -118, -123, -156, -157, -167, and -189) and 12 dioxin-like [DL-] PCBs was also analyzed in placenta samples [19]. All PCDD/F congeners tested, with the exception of 1,2,3,7,8,9-HxCDF, were found in all study samples. Toxic equivalent (TEQ) concentrations of PCDD/ Fs and DL-PCBs were $6.9 \mathrm{pg}$ WHO-TEQ/g lipid and $2.1 \mathrm{pg}$ WHO-TEQ/g lipid, respectively, with the most abundant $\mathrm{PCDD} / \mathrm{F}$ congeners being those with the longest half-lives [21].

\section{The Complexity of EDC Risk Assessment}

Risk assessment in the endocrine-disruption field is a highly complex task. For instance, dose-response assessment is usually based on the assumption of monotonic dose-response curves for almost all chemicals, with linear dose-response models predicting effects at low doses by extrapolation from effects at high doses [22]. However, this model is not generally suitable for the study of EDCs, because their hormone-like behavior can generate non-monotonic (e.g., U-shaped or other non-linear) doseresponse curves, in which effects can be more harmful at lower than at higher doses. This behavior is attributable to the complex dynamism that characterizes the occupation-saturation of the hormonal receptor [6, 23•]. Moreover, some EDCs can show a monotonic linear response when examined in a simple experimental test but can exhibit other response patterns in the presence of endogenous hormones; hence, their effects may depend on the hormonal status of an individual at the time of exposure [6]. These characteristics, which seem unique to EDCs, make their study particularly challenging. Moreover, the action of EDCs on a given tissue may vary according to their isoform and the presence or abundance of specific receptors on the tissue. Hence, realistic risk estimations must take account of the hormonal pattern of individuals, the susceptibility of each tissue or organ, and the timing of exposure, because small variations can affect the functionality of the system if homeostatic mechanisms are not adequate [7,8].

As shown above, mothers and their children are not exposed to a single chemical alone but rather to a complex mixture of compounds. Therefore, investigation of the impact on human health of EDC exposure should not be limited to the individual effects of single agents but should rather consider the cumulative effects of multiple chemicals [24, 25]. Several authors have pointed out that even the most comprehensive chemical analysis may only explain part of the biologically effective endocrine-disrupting potential evidenced in bioassays [26, 27]. For this reason, studies on environmental exposure during pregnancy and childhood have recommended the use of biomarkers to evaluate mixtures of EDCs in the organism as well as inter-individual variability [28]. Thus, our research group developed the total effective xenoestrogen burden (TEXB) as a reliable biomarker of the cumulative exposure to mixtures of xenoestrogens in the organism, using a specific bioassay to establish the combined estrogenicity in different biological samples $[26,28]$. TEXB is based on the proliferative effect on human breast cancer cells of human biological extracts, which has been applied to epidemiological studies in relation to the risk of developing several human diseases, including anomalies of sexual maturation in males, breast cancer, and type 2 diabetes [9, 29, 30]. In addition, an association has been found between higher prenatal TEXB levels and increased birth weight in males [31]. The use of TEXB in placenta samples appears to be a reasonable strategy for assessing maternal exposure to xenoestrogens and for estimating exposure of the fetus, and it may represent a more useful approach than the study of individual chemical residues $[9,26]$.

\section{Endocrine Disruptors and Human Diseases}

In 2002, the WHO Global Assessment of the State-of-theScience of Endocrine Disruptors report (WHO, 2002) summarized the current state of scientific knowledge on the potential effects of exposure to EDCs in humans and wildlife [32]. It was clear that certain environmental chemicals can interfere with normal hormonal processes, and progress has subsequently been made in the identification and quantification of a wide array of chemicals with endocrine-active properties. Evidence on the adverse health effects of EDC exposure was weak in the WHO 2002 report but is much stronger now, due to a shift in focus over the past decade from investigating associations between adult EDC exposure and disease outcomes $\left[2,33^{\circ}\right]$ to exploring associations between developmental exposures and disease outcomes later in life $[2,34]$. It has been verified that an increase in certain adverse health effects in populations may be attributable to exposure to EDCs. However, human studies can reveal associations but they cannot establish causal relationships, and it may never be possible to be absolutely certain that a specific exposure is responsible for a specific disease or dysfunction, due to the complexity of exposures and disease etiology across the lifespan (WHO, 2013) [2]. Nevertheless, some new prospective birth cohort studies have published suggestive results with regard to exposure-effect relationships [35, 36], including various human health concerns. A few examples are summarized below. 


\section{A. Testicular Dysgenesis Syndrome (TDS)}

Over the past few decades, most Western countries have reported an increase in male reproductive disorders, especially those related to TDS, including cryptorchidism (absence of one or both testes from the scrotum), hypospadias (abnormally placed urinary meatus), poor semen quality, and testicular cancer [37]. Although the etiology of TDS is not known in most cases, it has been hypothesized that this rise is due not only to improvements in clinical detection but also to a combination of genetic and environmental factors, including exposure to mixtures of environmental pollutants [38, 39•].

Several studies have identified a large group of EDCs that are able to interact with androgen receptors and therefore exert anti-androgenic effects [40, 41]. They include OCs, alkylphenols, parabens, brominated flame retardants, and PCBs, to which the general population is typically exposed on a daily basis [40, 41]. In fact, there is epidemiological evidence that human exposure to low doses of EDCs might be associated with urogenital tract malformations and decreased fertility [9, 42]. For instance, as part of research conducted in a mother-child cohort in Southern Spain (INMA Granada), we found an increased risk for male urogenital malformations in offspring in a nested case-control study, which was attributable to the combined effect of environmental estrogens assessed in placenta [9]. Evidence remains weak on the involvement in TDS genesis of single chemicals at current environmental levels. Animal studies have also shown a clear connection between in utero exposure to EDCs and adverse effects on male reproduction [43, 44].

\section{B. Obesity and Diabetes}

Recent studies in adults have linked EDC exposure to obesity, cardiovascular disease, diabetes, and the metabolic syndrome, diseases whose incidence has been rising worldwide (Fig. 1) [2, 45, 46]. The marked increase in obesity, especially over the past 2 decades, is usually attributed to a poor diet and lack of exercise, although some 'obesogens', EDCs that interfere with endocrine signaling and affect hormonally regulated metabolic processes, have also been implicated [45]. To date, the scientific community has identified at least 20 obesogens that may cause long-term obesity through their ability to modify hormonal and neuronal signaling pathways [47]. All of these EDCs are lipid-soluble substances that can be stored in fat cells and disrupt tissue function. When these cells lose fat, obesogens escape into the bloodstream and facilitate weight recovery. Moreover, during brain development, the EDCs affect both the hypothalamus, which participates in feeding behaviors, and the thyroid gland, involved in the regulation of body metabolism [48].

Once more, assessment of the effect of EDCs on obesity and diabetes is a highly complex task, given the other risk

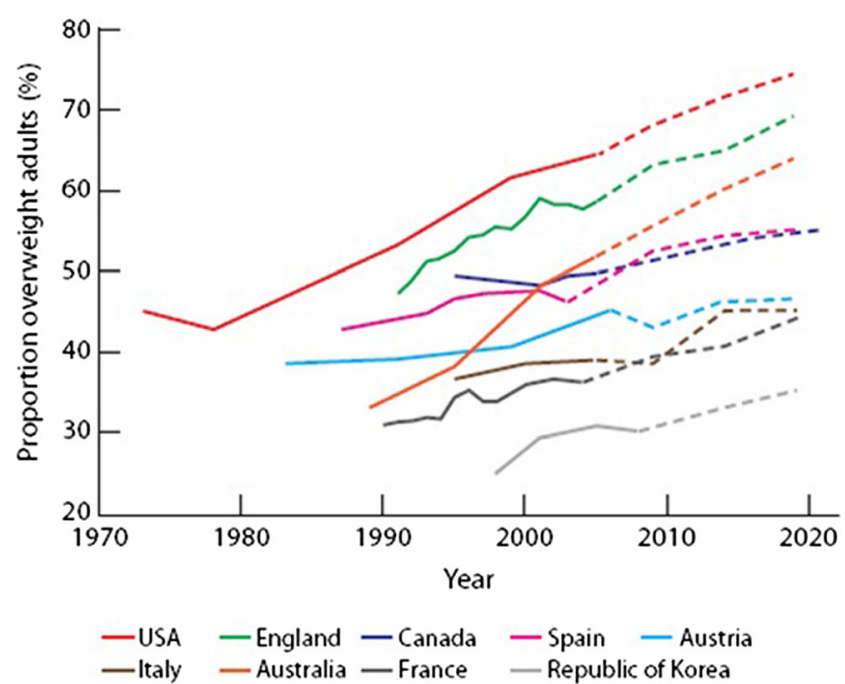

Fig. 1 Past (solid lines) and projected (dashed lines) overweight rates in selected OECD countries according to the OECD (OECD, 2010)

factors involved, e.g., diet and physical exercise (especially difficult to measure), and the need to evaluate of the effects of early life exposures to mixtures of EDCs on outcomes much later in life [49].

In parallel with the rise in obesity, the prevalence of type 2 diabetes worldwide is estimated to reach $4.4 \%$ in 2030 (vs. $2.8 \%$ in 2000) [50]. This increasing trend has been attributed to a combination of genetic and environmental factors [51], and chronic exposure to low doses of EDCs has also been implicated. Thus, exposure to certain persistent organic pollutants (POPs) has been associated with an increased risk of the disease [52-56], including recent findings from our group reporting a significant association between the adipose tissue concentrations of certain POPs and the risk of type 2 diabetes in an adult population [29]. Serum levels of POPs and/or PCBs have been positively associated with diabetes risk in cross-sectional and longitudinal studies and a meta-analysis [52-56], but the risks associated with individual POPs are not entirely consistent across studies.

The mechanisms involved may include potential synergisms between adipose tissue and POPs, epigenetic changes over generations, and a 'cocktail effect' of mixtures of POPs and/or other chemicals currently in use [29, 54]. This cocktail effect is a key point in considering the effects of long-term exposure to mixtures of POPs at low doses, including a possible immunotoxic effect [57]. This mechanism would induce a chronic low-grade inflammation process, reduced mitochondrial function and fatty acid oxidation, and increased lipolysis, which are all related to the insulin-resistance syndrome [58]. Animal models demonstrate that environmental doses of some EDCs produce insulin resistance and other altered metabolic parameters in adult mice. Moreover, experiments in rodents demonstrate that a brief exposure to some EDCs, such as BPAs, during pregnancy produces a 
prediabetic state in the mothers several months after labor and induces glucose intolerance, insulin resistance, and hyperinsulinemia in male offspring during adulthood. Additionally, molecular evidence indicates, at least for those EDCs with estrogenic activity, that estrogen receptors are involved in the induction of insulin resistance and the alteration of pancreatic $\beta$-cell function [59].

\section{Breast Cancer}

The incidence of breast cancer has increased to unprecedented levels worldwide over recent decades, and it is now the most frequent cancer among women in many parts of the world (Fig. 2) [60], suggesting that environmental factors play an important role in its etiology. Various epidemiological studies on environmental and occupational exposure have proposed certain xenoestrogens as potential candidates for the increase in breast cancer [61], and hundreds of these environmental chemicals from a range of dietary and domestic sources have been measured in human breast tissue [62, 63]. Investigations of associations between elevated levels of these compounds and the development of breast cancer have reported inconclusive results [61-70]; however, the lack of consistency among findings may be attributable to the numerous challenges faced by research on this issue. Thus, most studies investigated the effect of individual EDCs rather than addressing the environmental reality of long-term low-dose exposure to complex mixtures, also missing possible interactions between chemicals and physiological hormones, which may act together to alter the internal homeostasis of the estrogenic environment of breast tissue [26, 71].

Biomarkers based on hormonal activity have been developed for a more accurate quantification of exposure, allowing evaluation of the effects caused by cumulative exposure to a mixture of EDCs. In this regard, a statistically significant relationship was found between breast cancer risk and the combined effect of environmental xenoestrogens by applying a biomarker of exposure to assess the TEXB in human adipose tissue in a casecontrol study [30]. Environmental estrogens were separated from endogenous estrogens, and the combined estrogenic effect due to the bioaccumulation of organohalogenated xenoestrogens was determined from its proliferative effect in the E-screen assay [72].

To add further complication to the issue, breast cancer starts to develop many years before the onset of symptoms, and chemicals should be measured at multiple developmental time points. The chemical burden can also vary as a function of changes in lifestyle. Hence, both chemical analyses and studies of chemical burden are warranted in follow-up studies of women exposed to multiple residues in order to elucidate the clinical significance of the distribution of residues at low and high

Female breast cancer incidence per 100000

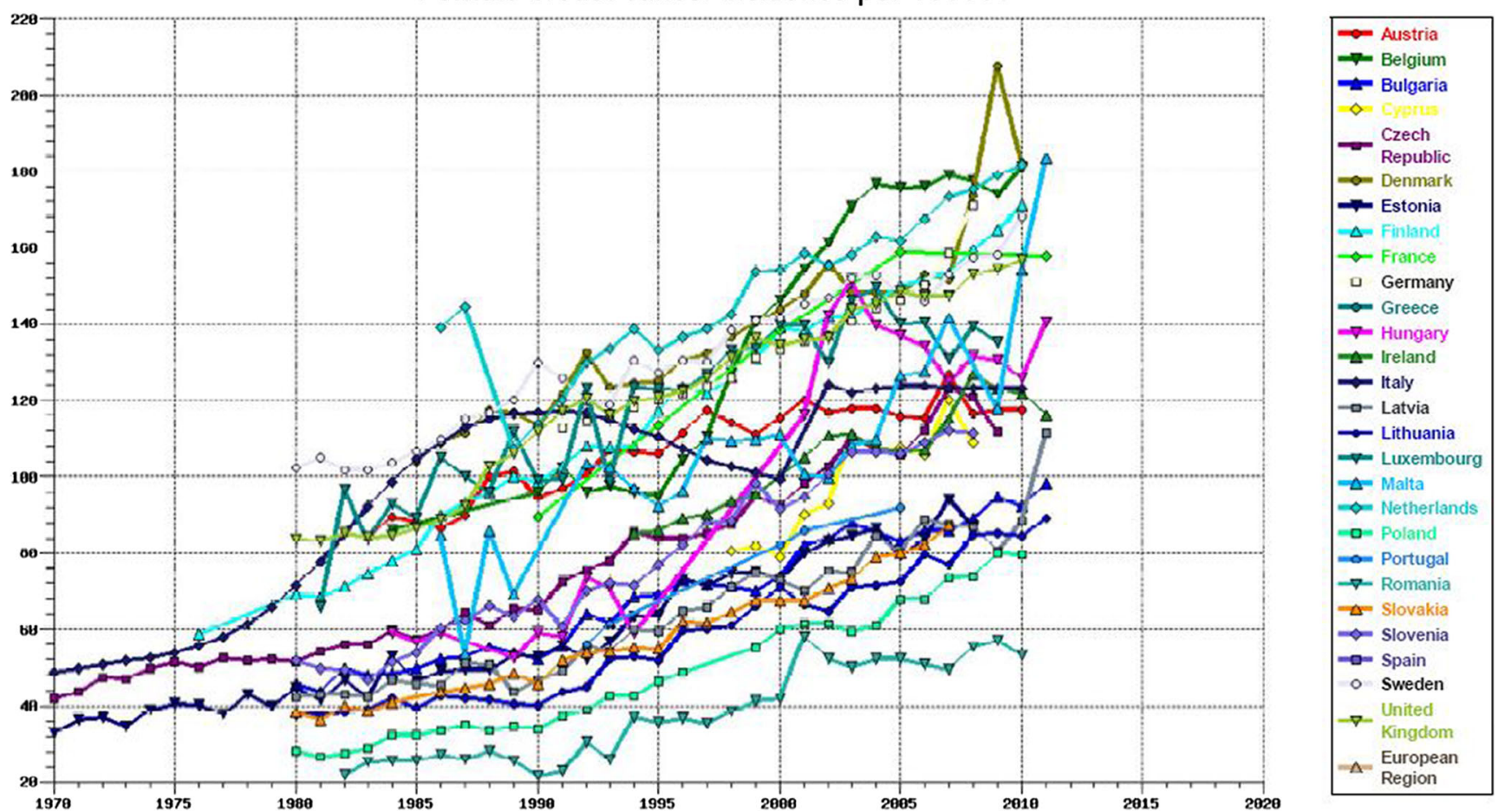

Fig. 2 Trends in breast cancer incidence in 27 European countries. Analysis of the European health for all database (online database). Copenhagen, WHO Regional Office for Europe, 2014 (http://data.euro.who.int/hfadb/) 
doses in the same individual. These types of study are essential to address current uncertainties about the functionality of EDCs in human tissues [73, 74].

\section{Public Policy Implications}

There is considerable political debate about the need for measures to reduce or avoid exposure to EDCs, and this controversy has affected the progress of knowledge on endocrine disruption. Thus, implementation of an action plan developed by the European Parliament in 1999 was limited to the prohibition of phthalates in pacifiers/teethers/nipples and bisphenol A in polycarbonate baby bottles, based on the precautionary principle to protect children's health. Our data show that Spanish children are in the top ranking of exposure to phthalates compared with other USA studies involving pregnant women [10], and we believe that the scientific evidence is adequate to support the implementation and extension of these preventive measures.

Growing demands for action to protect the public from EDCs led the Committee on Environment, Public Health and Food Safety of the European Parliament to propose a resolution, passed in 2012 [2012/2066(INI)], on the need to reduce human exposure to these chemicals, especially among the most vulnerable groups, taking into account the difficulty of establishing safe levels of exposure. It proposed improvements in the European regulation system - REACH - and the examination of all available scientific studies in a review of the risk-assessment process. As with REACH in the EU, a new chemicals policy in the USA has the potential to fuel global demand for safer substances and processes, increasing the incentive for research and development in green chemistry while improving human and environmental health [75]. In addition, environmental health scientists have an essential role in identifying and addressing the research questions that will arise with the development of new EDC policies. Scientists and representatives of environmental organizations recently published a report on key scientific issues relevant to the identification of EDCs for use in different regulatory contexts [76]. Some toxicologists involved with regulatory bodies, all editors of toxicology journals, recently declared in an open letter that the recommendations of the report were inadequate and that the current system of risk evaluation should be maintained [77]. However, a large group of scientists reaffirmed, in an editorial responding to the letter, that there was already sufficient evidence to support further measures to reduce EDC exposure [78].

While awaiting the implementation of stricter regulation of exposure to EDCs, health professionals should be fully aware of this issue, recognizing exposure and advising the population about the health risks. It is also important for healthcare professionals to use their influence in the policy-making arena to ensure that emerging scientific findings are translated into prevention-oriented action on a large scale [79]. It is time to act, identifying and reducing exposure to toxic environmental agents while addressing its consequences.

\section{Conclusion}

Increasing evidence supports the role of EDCs on human health. An important advance in recent years has been the increased number of prospective birth cohort studies revealing suggestive exposure-effect relationships. Consistent with the precautionary principle for reducing risk in the absence of causal evidence, the Royal College of Obstetricians and Gynecologists concluded, "Despite uncertainty surrounding the effect of common environmental chemicals, mothers should be made aware of the sources and routes of exposure, the potential risk to the baby and the important role that the mother can play in minimizing her baby's chemical exposure" [16]. Essential strategies for diminishing harmful EDC exposure and improving health outcomes are urgently needed.

Acknowledgments This work was supported by grants from the Spanish Ministry of Health (FIS-PI11/00610); Instituto de Salud Carlos III (Red INMA G03/176 and CB06/02/0041); the EU Commission (QLK41999-01422, QLK4-2002-00603, and CONTAMED FP7-ENV-212502), the Consejería de Salud de la Junta de Andalucía (grant numbers 183/07 and 0675/10). The HUSC BioBank, integrated in the Andalusia Public Health System (SSPA) and the National Biobank Network, is financed by the Institute of Health Carlos III (project RD09/0076/00148), and the Regional Government of Andalucía.

\section{Compliance with Ethics Guidelines}

Conflict of Interest Mariana F. Fernández, Marta Román, Juan Pedro Arrebola, and Nicolás Olea declare that they have no conflicts of interest.

Human and Animal Rights and Informed Consent This article contains data from studies with human subjects, some of them performed by the authors. All those studies followed the guidelines laid down in the Declaration of Helsinki and were approved by the Ethics Committee of the corresponding Institutions.

\section{References}

Papers of particular interest, published recently, have been highlighted as:

- Of importance

1. Reinen J et al. Endocrine disrupting chemicals-Linking internal exposure to vitellogenin levels and ovotestis in Abramisbrama from Dutch surface waters. Environ Toxicol Pharmacol. 2010;30(3):209-23.

2. Bergman A et al. State of the science of endocrine disrupting chemicals. 2013. http://www.who.it/ceh/publications/endocrine/ en. Accessed 04 October 2013. 
3. Olea $\mathrm{N}$ et al. Perspectivas en disrupción endocrina. Gac Sanit. 2002;16:250-6.

4. Ropero $\mathrm{AB}$ et al. Rapid endocrine disruption: environmental estrogen actions triggered outside the nucleus. J Steroid Biochem Mol Biol. 2006;102(1-5):163-9.

5. Anway MD, Skinner MK. Epigenetic programming of the germ line: effects of endocrine disruptors on the development of transgenerational disease. Reprod Biomed Online. 2008;16:23-5.

6. Vandenberg LN et al. Regulatory decisions on endocrine disrupting chemicals should be based on the principles of endocrinology. Reprod Toxicol. 2013;38:1-15.

7. Schug TT et al. Endocrine disrupting chemicals and disease susceptibility. J Steroid BiochemMol Bio. 2011;127:204-15.

8. Diamanti-Kandarakis $\mathrm{E}$ et al. The impact of endocrine disruptors on endocrine targets. Horm Metab Res. 2010;42:543-52.

9. Fernandez MF et al. Human exposure to endocrine-disrupting chemicals and prenatal risk factors for cryptorchidism and hypospadias: a nested case-control study. Environ Health Perspect. 2007;115:8-14.

10. Casas $\mathrm{L}$ et al. Urinary concentrations of phthalates and phenols in a population of Spanish pregnant women and children. Environ Int. 2011;37(5):858-66.

11. Cerrillo I et al. Endosulfan and its metabolites in fertile women, placenta, cord blood, and human milk. Environ Res. 2005;98:233-9.

12. Barouki $\mathrm{R}$ et al. Developmental origins of non-communicable disease: implications for research and public health. Environ Health. 2012;27:42.

13. Iyengar GV, Rapp A. Human placenta as a 'dual' biomarker for monitoring fetal and maternal environment with special reference to potentially toxic trace elements. Part 2: essential minor, trace and other (non-essential) elements in human placenta. Sci Total Environ. 2001;280(1-3):207-19.

14. Guxens $\mathrm{M}$ et al. Cohort profile: the INMA-INfancia y Medio Ambiente-(Environment and Childhood) Project. Int J Epidemiol. 2012;41(4):930-40.

15. Woodruff TJ et al. Environmental chemicals in pregnant women in the United States: NHANES 2003-2004. Environ Health Perspect. 2011;119(6):878-85.

16. Stotland NE et al. Counseling patients on preventing prenatal environmental exposures - a mixed-methods study of obstetricians. PLoS One. 2014;9(6):e98771.

17. Lopez-Espinosa MJ et al. Organochlorine pesticides in placentas from Southern Spain and some related factors. Placenta. 2007;28(7):631-8.

18. Jiménez-Díaz I et al. Determination of bisphenol A and its chlorinated derivatives in placental tissue samples by liquid chromatography-tandem mass spectrometry. J Chromatogr B Analyt Technol Biomed Life Sci. 2010;878(32):3363-9.

19. Jiménez-Díaz I et al. A new liquid chromatography-tandem mass spectrometry method for determination of parabens in human placental tissue samples. Talanta. 2011;84(3):702-9.

20. Vela-Soria $F$ et al. Determination of benzophenones in human placental tissue samples by liquid chromatography-tandem mass spectrometry. Talanta. 2011;85(4):1848-55.

21. Fernandez MF et al. Levels of polychlorinated dibenzo-p-dioxins, dibenzo furans and dioxin-like polychlorinated biphenyls in 50 placentas from the Spanish INMA birth cohort study. Sci Total Environ. 2012;441:49-56.

22. Welshons WV et al. Large effects from small exposures. I. Mechanisms for endocrine-disrupting chemicals with estrogenic activity. Environ Health Perspect. 2003;111(8):994-1006.

23. Vandenberg LN et al. Hormones and endocrine-disrupting chemicals: low-dose effects and nonmonotonic dose responses. Endocr Rev. 2012;33:378-455. This review article provides hundreds of examples showing that non-monotonicity and low-dose effects are common in studies of hormones and EDCs.
24. Payne J et al. Prediction and assessment of the effects of mixtures of four xenoestrogens. Environ Health Perspect. 2000;108(10):983-7.

25. Rajapakse N, Silva E, Kortenkamp A. Combining xenoestrogens at levels below individual no-observed-effect concentrations dramatically enhances steroid hormone action. Environ Health Perspect. 2002;110(9):917-21.

26. Fernández MF et al. Assessment of total effective xenoestrogen burden in adipose tissue and identification of chemicals responsible for the combined estrogenic effect. Anal Bioanal Chem. 2004;379: $163-70$.

27. Rasmussen TH et al. Assessment of xenoestrogenic exposure by a biomarker approach: application of the E-Screen bioassay to determine estrogenic response of serum extracts. Environ Health. 2003;2(1):12.

28. Lopez-Espinosa MJ et al. Assessment of the total effective xenoestrogen burden in extracts of human placentas. Biomarkers. 2009;14(5):271-7.

29. Arrebola JP et al. Adipose tissue concentrations of persistent organic pollutants and prevalence of type 2 diabetes in adults from Southern Spain. Environ Res. 2013;122:31-7.

30. Ibarluzea JM et al. Breast cancer risk and the combined effect of environmental estrogens. Cancer Causes Control. 2004;15:591-600.

31. Vilahur $\mathrm{N}$ et al. Male specific association between xenoestrogen levels in placenta and birthweight. Environ Int. 2013;51:174-81.

32. WHO. Global assessment of the state of-the-science of endocrine disruptors. 2002. http://www.who.int/ipcs/publications/new issues/ endocrine_disruptors/en. Accessed 04 October 2013.

33. Thayer KA et al. Role of environmental chemicals in diabetes and obesity: a National Toxicology Program workshop review. Environ Health Perspect. 2012;120(6):779-89. This review article provides a thorough review of the role of environmental chemicals in diabetes.

34. Grandjean P et al. The Faroes statement: human health effects of developmental exposure to chemicals in our environment. Basic Clin Pharmacol Toxicol. 2008;102(2):73-5.

35. Casas $\mathrm{M}$ et al. Exposure to brominated flame retardants, perfluorinated compounds, phthalates and phenols in European birth cohorts: ENRIECO evaluation, first human biomonitoring results, and recommendations. Int J Hyg Environ Health. 2013;216(3):230-42.

36. Larsen PS et al. Pregnancy and birth cohort resources in Europe: a large opportunity for aetiological child health research. Paediatr Perinat Epidemiol. 2013;27(4):393-414.

37. Skakkebaek NE, Rajpert-De Meyts E, Main KM. Testicular dysgenesis syndrome: an increasingly common developmental disorder with environmental aspects. Hum Reprod. 2001;16(5):972-8.

38. Main KM et al. Genital anomalies in boys and the environment. Best Pract Res Clin Endocrinol Metab. 2010;24(2): 279-89.

39. Juul A et al. Possible fetal determinants of male infertility. Nat Rev Endocrinol. 2014;10(9):553-62. This review article discusses the current knowledge of a range of male reproductive health problems and focusses in particular on the developmental origin of testicular pathologies.

40. Jiménez-Díaz I et al. Simultaneous determination of the UV-filters benzyl salicylate, phenyl salicylate, octyl salicylate, homosalate, 3(4-methylbenzylidene) camphor and 3-benzylidene camphor in human placental tissue by LC-MS/MS. Assessment of their in vitro endocrine activity. J Chromatogr B Analyt Technol Biomed Life Sci. 2013;936:80-7.

41. Molina-Molina JM et al. In vitro study on the agonistic and antagonistic activities of bisphenol-S and other bisphenol-A congeners and derivatives via nuclear receptors. Toxicol Appl Pharmacol. 2013;272(1):127-36.

42. Mendiola J et al. Sperm counts may have declined in young university students in Southern Spain. Andrology. 2013;1(3):408-13. 
43. Welsh $\mathrm{M}$ et al. Identification in rats of a programming window for reproductive tract masculinization, disruption of which leads to hypospadias and cryptorchidism. J Clin Invest. 2008;118(4):1479-90.

44. Christiansen $\mathrm{S}$ et al. Combined exposure to anti-androgens causes markedly increased frequencies of hypospadias in the rat. Int J Androl. 2008;31(2):241-8.

45. Zhang $P$ et al. Global healthcare expenditure on diabetes for 2010 and 2030. Diabetes Res Clin Pract. 2010;87(3):293-301.

46. Taylor KW et al. Evaluation of the association between persistent organic pollutants (POPs) and diabetes in epidemiological studies: a national toxicology program workshop review. Environ Health Perspect. 2013;121(7):774-83.

47. Grun F, Blumberg B. Perturbed nuclear receptor signaling by environmental obesogens as emerging factors in the obesity crisis. Rev Endocr Metab Disord. 2007;8(2):161-71.

48. Tremblay A et al. Thermogenesis and weight loss in obese individuals: a primary association with organochlorine pollution. Int $\mathrm{J}$ Obes Relat Metab Disord. 2004;28(7):936-9.

49. Longnecker MP. Pharmacokinetics variability and the miracle of modern analytical chemistry. Epidemiology. 2006;17(4):350-1.

50. Wild et al. Global prevalence of diabetes: estimates for the year 2000 and projections for 2030. Diabetes Care. 2004;27:1047-53.

51. Das UN. Obesity: genes, brain, gut, and environment. Nutrition. 2010;26:459-73.

52. Airaksinen $\mathrm{R}$ et al. Association between type 2 diabetes and exposure to persistent organic pollutants. Diabetes Care. 2011;34:1972-9.

53. Everett CJ, Matheson EM. Biomarkers of pesticide exposure and diabetes in the 1999-2004 national health and nutrition examination survey. Environ Int. 2010;36:398-401.

54. Lee DH et al. Polychlorinated biphenyls and organochlorine pesticides in plasma predict development of type 2 diabetes in the elderly: the prospective investigation of the vasculature in Uppsala Seniors (PIVUS) study. Diabetes Care. 2011;34:1778-84.

55. Wu $\mathrm{H}$ et al. Persistent organic pollutants and type 2 diabetes: a prospective analysis in the nurses' health study and meta-analysis. Environ Health Perspect. 2013;121(2):153-61.

56. Gasull AJ et al. Blood concentrations of persistent organic pollutants and pre-diabetes and diabetes in the general population of Catalonia. Environ Sci Technol. 2012;46:7799-810.

57. Wang et al. Acceleration of autoimmunity by organochlorine pesticides: a comparison of splenic B-cell effects of chlordecone and estradiol in (NZBxNZW)F1 mice. Toxicol Sci. 2007;99:141-52.

58. Guilherme A et al. Adipocyte dysfunctions linking obesity to insulin resistance and type 2 diabetes. Nat Rev Mol Cell Biol. 2008;9:367-77.

59. Alonso-Magdalena P, Quesada I, Nadal A. Endocrine disruptors in the etiology of type 2 diabetes mellitus. Nat Rev Endocrinol. 2011;7(6):346-53.

60. Stuckey A. Breast cancer: epidemiology and risk factors. Clin Obstet Gynecol. 2011;54:96-102.

61. Olea N, Fernandez M. Endocrine disruption. J Epidemiol Commun Health. 2007;61:372-3.

62. Darbre PD, Charles AK. Environmental oestrogens and breast cancer: evidence for a combined involvement of dietary, household and cosmetic xenoestrogens. Anticancer Res. 2010;30:815-28.
63. Brody JG et al. Environmental pollutants and breast cancer. Cancer. 2007;109 Suppl 12:2667-711.

64. Cerrillo I et al. Environmental and lifestyle factors for organochlorine exposure among women living in Southern Spain. Chemosphere. 2006;62:1917-24.

65. Fernandez MF et al. PBDEs and PBBs in the adipose tissue of women from Spain. Chemosphere. 2007;66:377-83.

66. Fernandez MF et al. Polychlorinated biphenyls (PCBs) and hydroxy-PCBs in adipose tissue of women in Southeast Spain. Chemosphere. 2008;71:1196-205.

67. Lopez-Espinosa MJ et al. Dioxins in adipose tissue of women in Southern Spain. Chemosphere. 2008;73:967-71.

68. Lopez-Espinosa MJ et al. Nonylphenol and octylphenol in adipose tissue of women in Southern Spain. Chemosphere. 2009;76:84752.

69. Fernandez MF et al. Bisphenol-A and chlorinated derivatives in adipose tissue of women. Reprod Toxicol. 2007;24:259-64.

70. Darbre PD, Harvey PW. Paraben esters: review of recent studies of endocrine toxicity, absorption, esterase and human exposure, and discussion of potential human health risks. J Appl Toxicol. 2008;28:561-78.

71. Darbre PD, Fernandez MF. Environmental oestrogens and breast cancer: long-term low-dose effects of mixtures of various chemical combinations. J Epidemiol Commun Health. 2013;67(3):203-5.

72. Soto AM, Sonnenschein C, Chung KL, Fernandez MF, Olea N, Serrano FO. The E-SCREEN assay as a tool to identify estrogens: an update on estrogenic environmental pollutants. Environ Health Perspect. 1995;103 Suppl 7:113-22.

73. Porta M. Persistent toxic substances: exposed individuals and exposed populations. J Epidemiol Commun Health. 2004;58:534-5.

74. Haffner A, Schecter A. Persistent organic pollutants (POPs): a primer for practicing clinicians. Curr Environ Health Rep. 2014;1: 123-31.

75. Wilson MP, Schwarzman MR. Toward a new U.S. chemicals policy: rebuilding the foundation to advance new science, green chemistry, and environmental health. Environ Health Perspect. 2009;117(8):1202-9.

76. Munn S, Goumenou M. Report of the endocrine disrupters - Expert Advisory Group (ED EAG). Key scientific issues relevant to the identification and characterisation of endocrine disrupting substances. $2013 \mathrm{http}: / /$ ec.europa.eu/dgs/jrc/index.cfm?id=1410\&dt code=NWS\&obj id=16530\&ori=RSS. Accessed 04 October 2013.

77. Dietrich DR et al. Scientifically unfounded precaution drives European Commission's recommendations on EDC regulation, while defying common sense, well-established science and risk assessment principles. Chem Biol Interact. 2013;205:A1-5.

78. Bergman $\AA$ et al. The impact of endocrine disruption: a consensus statement about the state of the science. Environ Health Perspect. 2013;12:A104-6.

79. The American College of Obstetricians and Gynecologists. Committee Opinion 575.Exposure to toxic environmental agents. 2013. http://www.acog.org/Resources And Publications/ Committee Opinions/Committee on Health Care for Underserved Women/Exposure to Toxic Environmental Agents. Accessed 04October-2013. 